\title{
Adaptive beaconing for collision avoidance and tracking accuracy in vehicular networks
}

\author{
Long Sun ${ }^{1,2}$, Aiping Huang ${ }^{1,2}$, Hangguan Shan ${ }^{1,2 *},{\text { Lin } \mathrm{Cai}^{3}}^{3}$ \\ 1. College of Information Science and Electronic Engineering, Zhejiang University, Hangzhou 310027, China \\ 2. Zhejiang Provincial Key Laboratory of Information Processing and Communication Networks, \\ Hangzhou 310027, China
}

3. University of Victoria, Victoria BC V8W 3P6, Canada

* Corresponding author, Email: hshan@zju.edu.cn

\begin{abstract}
In vehicular networks, the exchange of beacons among neighboring vehicles is a promising solution to guarantee a vehicle's safety. However, frequent beaconing under high vehicle density conditions will cause beacon collisions, which are harmful to a vehicle's driving safety and the location tracking accuracy. We propose an ABIwRC (Adaptive Beaconing Interval with Resource Coordination) method for a highway scenario. Each vehicle broadcasts beacon interval requests, including the intervals needed for both the vehicle's driving safety and location tracking accuracy. The RSU(Road Side Unit) allocates resources for a vehicle's beaconing according to the requests from all vehicles and the interference relationship between the vehicles in adjacent RSUs. We formulate a resource allocation problem for maximizing the sum utility, which measures the satisfaction of vehicles' requests. We then transform the optimization problem into a maximum weighted independent set problem, and propose an algorithm to solve this efficiently. Simulation results show that the proposed method outperforms the benchmark in terms of beacon reception ratio, vehicle driving safety, and location tracking accuracy.
\end{abstract}

Keywords: vehicular networks, beacon, driving safety, location tracking accuracy, maximum weight independent set, interference avoidance

\section{Introduction}

As an important part of ITSs (Intelligent Transportation Systems), vehicular networks are promising to provide not only convenience and infotainment ${ }^{[1-5]}$, but also improved driving safety and transportation efficiency ${ }^{[6,7]}$. One important way to guarantee a vehicle's driving safety is periodic beaconing ${ }^{[8,9]}$. A vehicle can become aware of its driving environment from the beacon messages received from other vehicles in the vicinity. However, when the number of vehicles is large and/or the available radio resources are insufficient, frequent beaconing can cause beacon collisions; these can result in a low beacon message reception ratio or high latency, and thus lead to possible traffic accidents. Adopting less frequent beaconing can reduce collisions, but may not always be suitable. Because a larger beacon interval reduces the timeliness of the beacon message, and a vehicle's driving

Manuscript received Jan. 27, 2017; accepted Mar. 25, 2017

This work is supported in part by the Zhejiang Provincial Public Technology Research of China (No. 2016C31063), the Fundamental Research Funds for the Central Universities (No. 2015XZZX001-02), a research grant from the Natural Sciences and Engineering Research Council of Canada. 
safety and location tracking accuracy may be negatively impacted. Moreover, vehicles located in the coverage area of different RSUs (Road Side Units) may be allocated the same time and frequency for beaconing, and thus their beacon messages may collide, which affects the performance of the beaconing service. If the RSUs need to coordinate resource allocation among themselves, unordered coordination may lead to message collision. To address the above problems, it is necessary to study the resource coordination among RSUs and the vehicles' adaptive beaconing method together.

Existing researches have proposed adaptive beaconing methods aimed at increasing the beacon reception ratio and/or location tracking accuracy in vehicular networks ${ }^{[10-13]}$. In Ref. [10], the location deviation was adopted as a measure for vehicles to generate beacon messages. In Refs. [11] and [12], vehicles adapt beacon rates according to the state of the communication environment (i.e., the channel congestion conditions, packet delivery ratio, and average beacon inter-reception time) and the vehicle's driving state (i.e., speed, and the number of neighboring vehicles). In Ref. [13] an adaptive neighbor item expiration time and beacon rate scheme was proposed in order to improve the awareness of the surrounding vehicles according to their position, speed, and orientation. However, these studies adopted the CSMA/CA (Carrier Sense Multiple Access with Collision Avoidance) mechanism to access the channel, which could result in potential collisions due to hidden terminals. In Ref. [14], a RBEM/CBEM (Readyto-Broadcast-Emergency-Message and Clear-toBroadcast-Emergency-Message) based handshake mechanism was proposed to reserve a channel and to increase the packet reception ratio. However, the beaconing latency and high beacon reception probability still cannot be guaranteed when the vehicle density is high. To improve the safety message reliability and to ensure bounded delay, conflict-free MAC (Medium Access Control) protocols, such as TDMA (Time-Division Multiple-Access) ${ }^{[15,16]}$ and SDMA (Space-Division Multiple-Access)/LDMA
(Location-Division Multiple-Access) $^{[17,18]}$, have been proposed in the literatures. In Ref. [16], a time slotbased mechanism to access channels was proposed, which utilized a multi-rate beacon transmission time control mechanism to mitigate channel congestion. In Ref. [17], the road is divided into segments, and in each segment a control vehicle allocates slots to the other vehicles. However, these researches are aimed at improving the network metrics (i.e., the channel occupation ratio, and packet reception ratio), and cannot naturally improve traffic metrics like vehicle driving safety and location tracking accuracy.

In this paper, we study adaptive beaconing for vehicle driving safety and location tracking accuracy in vehicular networks in a highway scenario. To define the vehicle's state and driving environment, we define two slot intervals in which the vehicle requests broadcast of a beacon message (abbreviated to BI (Beacon Interval) request) to guarantee the vehicle's driving safety and location tracking accuracy, respectively. To reduce the delay or loss of beacon messages due to a collision, we adopt the RSUs as the central controllers for allocating radio resources. To avoid the collisions of beacon messages from vehicles located in adjacent RSUs, we let the RSUs conduct resource coordination with their adjacent RSUs. To achieve a reasonable allocation, we define a utility function with the BI requests and the number of beaconing slots that the vehicle has waited for as variables. We formulate the resource allocation as a utility maximization problem and transform it into an MWIS (Maximum Weighted Independent Set) problem, whereby a heuristic algorithm is proposed to efficiently solve the resource allocation problem. Simulation results show that the proposed method outperforms the baseline algorithms in terms of beacon reception ratio, safety, and tracking accuracy.

Our contributions are summarized as follows.

- We propose a coordination mechanism among RSUs. This mechanism enables RSUs to coordinate resource usage with adjacent RSUs in an orderly and fair manner, thus preventing vehicles from being allocated the same resource, and avoiding the collision of beacon messages from vehicles in adjacent RSUs. 


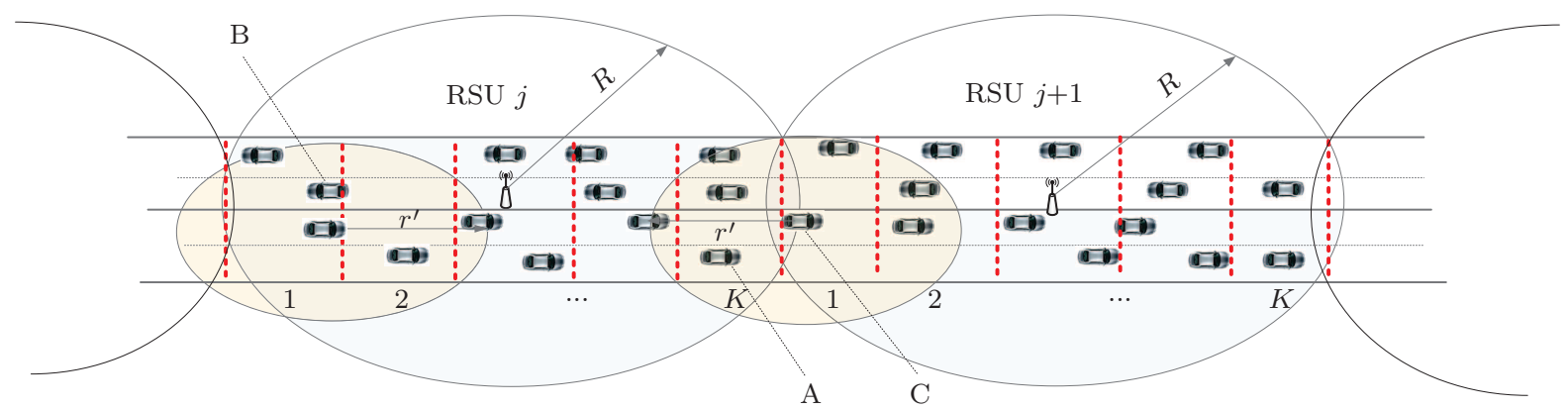

Figure 1 Scenario

- We define two BI requests to represent the maximal beacon slot intervals that guarantee safety and location tracking accuracy. The former is a function of the time headway, which is a measure of crash probability. The latter is a function of the acceleration variation, which reflects the vehicle's driving state.

- We define a utility function as a basis of resource allocation. This is a function of the BI requests and the number of beaconing slots that a vehicle has waited for, and thus is the achievable level of satisfaction of BI requests when the vehicle is allocated a certain resource block.

- We formulate the resource allocation into an optimization problem. Both the interference relationship among vehicles and the resource allocation constraints are considered, so that the resource allocation can avoid beacon collisions and fulfill vehicle requests as much as possible.

- We propose a method to transform the optimization problem into a maximum weighted independent set problem, which facilitates problem solving. The method maps the combinations of vehicles and resource blocks, the constraint relationship and interference relationship, and the utility value that a vehicle obtains when it is allocated a certain resource block to the vertices, edges, and weights in a directed weighted graph, respectively.

The remainder of the paper is organized as follows. Section 2 presents the system model. In section 3 , the adaptive beaconing interval with resource coordination method for vehicle crash avoidance and location tracking accuracy is proposed. The resource coordination is designed, and the resource allocation is formulated as a utility maximization problem. To solve the problem, a heuristic algorithm is developed in section 4 . In section 5 , simulation results are presented and discussed. The conclusions of this study are presented in section 6 .

\section{System model}

A bidirectional highway with a line topology is considered, as shown in Fig. 1. RSUs are deployed along the road, and are indexed sequentially; their signals can cover the whole road. The RSUs are connected through backhaul, and thus can exchange information. We divide the road within each RSU's transmission range into $K$ road segments each with a length of $d=2 R / K$, where $R$ is the transmission range of an RSU. The number of vehicles within RSU $j$ 's transmission range is denoted as $V^{j}$. The number of vehicles within road segment $k$ in $\mathrm{RSU} j$ is denoted as $V_{k}^{j}$, and thus $\sum_{k=1}^{K} V_{k}^{j}=V^{j}$. Each vehicle is assumed to be equipped with two antennas which help the vehicle to send and receive signals in different frequency bands simultaneously. The transmission range of any vehicle is $r(<R)$. The interference range of any vehicle denoted by $r^{\prime}$ is assumed to be no less than $r$ but less than $R$, due to different RSU and vehicle transmission power levels. The assumption that $r^{\prime}<R$ helps us simplify the presentation of the proposed method, but this can be easily extended to a more generalized case. The unit disk channel model ${ }^{[19]}$ is assumed, where two nodes can communicate successfully with each other using a fixed transmission rate if and only if their distance 


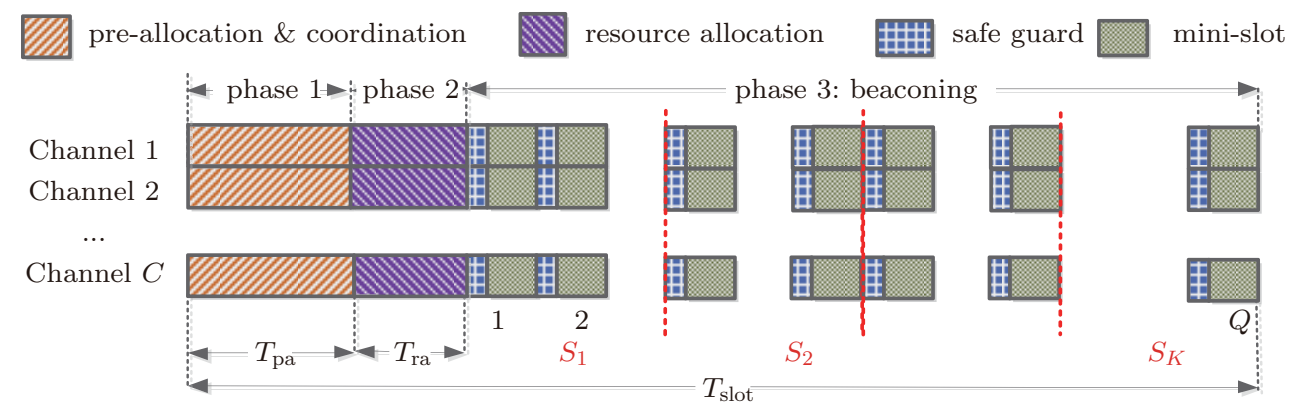

Figure 2 Slot structure

is smaller than the transmitter's transmission range, and any other nodes transmitting simultaneously are at a distance of $r^{\prime}$ from the receiver.

Each RSU maintains a "vehicle list" to store the vehicles' basic information from the last slot, and each column in the list corresponds to a vehicle within the RSU's transmission range. Each vehicle maintains a "neighbor list" to store its neighbors' basic information. The basic information for any vehicle comprises five parameters: identification ID, location $L$, velocity $w$, acceleration $\alpha$, and heading $h$.

The radio resource is partitioned into $C$ orthogonal dedicated channels and a service channel, and the transmission is time slotted. The RSUs and vehicles are able to listen to all of the dedicated $C$ channels simultaneously. The service channel is for vehicles newly entering the coverage area of an RSU to inform the RSU of their basic information. The duration of a slot is $T_{\text {slot }}$ seconds. The structure of the dedicated channels is shown in Fig. 2. Each slot consists of a pre-allocation phase with a duration of $T_{\mathrm{pa}}$ seconds, a resource allocation phase with a duration of $T_{\text {ra }}$ seconds, and a beaconing phase of $T_{\text {slot }}-T_{\text {pa }}-T_{\text {ra }}$ seconds. The pre-allocation phase is used for the pre-calculation of radio resource allocation by each RSU, and for resource coordination among RSUs to avoid beacon collision. The resource allocation phase is used for each RSU to determine and broadcast the final resource allocation scheme in the slot. The beaconing stage contains $Q$ mini-slots and there are safe guards between them. Here $Q=$ $\left\lfloor\left(T_{\text {slot }}-T_{\text {pa }}-T_{\text {ra }}\right) /\left(F / B+T_{\text {guard }}\right)\right\rfloor$, where $\lfloor x\rfloor$ is the floor function, $B$ is the transmission rate, $F$ is the number of bits in a beacon message, and $T_{\text {guard }}$ is the duration of a safe guard. The $q$ th mini-slot in the $c$ th channel is called the RB (Resource Block) $S_{q, c}, q=1,2, \cdots, Q, c=1,2, \cdots, C$. Each RB can be used for one vehicle to broadcast a beacon message. We divide all RBs into $K$ non-overlapping RPs (Resource Pools) $S_{k}(k=1,2, \cdots, K)$, as shown in Fig. 2, where RP $S_{k}$ is reserved for vehicles located in road segment $k$. If we define set $S=S_{1} \cup S_{2} \cup \cdots \cup S_{K}$, then there are $Q C$ RBs in $S$.

The reason for reserving an $\mathrm{RP}$ for each road segment is to efficiently control the inter-cell interference from adjacent RSUs. Specifically, road segment $K$ in RSU $j$ is adjacent to road segment 1 in RSU $j+1$, and the two groups of vehicles located within the two road segments are close to each other, but will not interfere with each other because they use RBs in RPs $S_{K}$ and $S_{1}$, respectively (see vehicles A and $\mathrm{C}$ in Fig. 1). Moreover, the vehicles located in road segment $k$ in $\operatorname{RSU} j$ and those in $\operatorname{RSU} j+1$ all use RBs in RP $S_{k}$ if the distance between the two road segments (i.e., $2 R-d$ ) is larger than $2 r^{\prime}$. Their beacon messages will not collide because these two groups of vehicles are far away from each other, in the same way as vehicles B and $\mathrm{C}$ in Fig. 1.

The number of road segments $K$ should satisfy $K \geqslant R /\left(R-r^{\prime}\right)$, so as to satisfy $2 R-d \geqslant 2 r^{\prime}$ as mentioned above. The larger the value of $K$ is, the further away the road segments with the same index will be. This has benefits for collision avoidance. However, a larger $K$ means less RBs in each RP, which increases the probability of requiring resource coordination among RSUs, and thus increases the information exchange overhead. An appropriate value of $K$ should be selected to effect a good tradeoff. 


\section{Adaptive beaconing method for safety guarantee and location track- ing accuracy}

Vehicles need to timely and reliably broadcast beacon messages. A timely broadcast means that the BI is small enough to satisfy safety or location tracking accuracy requests. To reliably broadcast and avoid collision, beacon messages should be transmitted via RBs that are not overlapping in time or frequency dimensions, where the inter-vehicle distance is less than the interference range. This requires appropriate resource allocation. We divide the complicated resource allocation procedure involving multiple RSUs into two steps. In the first step, as described later in section 3.2, the RBs' usage is coordinated among adjacent RSUs; this ensures that the RPs used by adjacent road segments among adjacent RSUs (e.g., road segment $K$ in RSU $j$ and road segment 1 in RSU $j+1$ ) not only satisfy the BI requests of the vehicles within the road segments, but also do not overlap with each other. In the second step, as described later in section 3.4, each RSU allocates RBs in each RP to the vehicles located in the corresponding road segment.

\subsection{Communication procedure}

When a new vehicle drives into an RSU's coverage area, it will competitively access the service channel, for example, based on the CSMA/CA mechanism, and pass its basic information to the RSU. After receiving the new vehicle's basic information, the RSU sets up a new column in its "vehicle list" to store this information.

The RSUs and the vehicles located within their transmission range operate according to the slot structure shown in Fig. 2. In the pre-allocation and coordination phase (phase 1), the RSUs preallocate RBs according to the "vehicle list". If an RSU finds that the number of RBs in a certain RP is less than the number of vehicles that are in the corresponding road segment and need to broadcast beacon messages, the RSU will coordinate with its adjacent RSUs through backhaul to "borrow" RBs from the other RPs; this is described later in section 3.2. In the resource allocation phase (phase 2), the RSUs repartition the RPs given the "borrowed" RBs, allocate the RBs in each RP to the vehicles located in the corresponding road segment, and broadcast allocation results in each channel.

In the beaconing phase (phase 3 ), each vehicle that has been allocated an $\mathrm{RB}$ in the current slot generates its BI requests, adds the BI requests to its beacon message, and broadcasts the beacon message in the allocated RB. Meanwhile, all vehicles listen to other vehicles' broadcast on a receiving antenna, and then update the information in their "neighbor lists". A column in the RSU's "vehicle list" (or vehicle's "neighbor list") is updated for each slot using the vehicle's (neighbor's) recent information received in that slot; if the vehicle's (neighbor's) beacon message in the slot is not received, the estimated information is derived from the historical information stored in the column. If the RSU (vehicle) has not received a vehicle's (neighbor's) beacon message for $N_{0}$ slots, it assumes that the vehicle (neighbor) has driven out of its transmission range, and it therefore deletes the column from the list. Here, $N_{0}=\left\lfloor T_{0} / T_{\text {slot }}\right\rfloor$, and $T_{0}$ is the standard maximum allowed BI duration ${ }^{[20]}$.

\subsection{Coordination among RSUs}

In phase 1 , for any RP $S_{k}$, an RSU can "borrow" RBs from its other RPs when it finds that the number of RBs in $S_{k}$ is insufficient. However, if the vehicles located in road segment $k$ borrow and use RBs in any other RPs, their beacon messages may collide with those of the vehicles in the adjacent RSUs. Therefore, although borrowing takes place from one RSU's different RPs, the RSU should coordinate with its adjacent RSUs first. This coordination means that borrowing occurs similarly among RSUs. For the sake of brevity, the RSU that proposes the borrowing request (e.g., a request to borrow $\mathrm{RBs}$ for $\mathrm{RP}$ $S_{k}$ ) is called the requester, while the RSU that coordinates responses (i.e., permits the "lending" of RBs from the other RPs) is called the responder. 
The coordination procedure is shown in Fig. 3. First, the requester sends the borrowing request which contains the required number of RBs of certain RPs (e.g., $S_{k}$ ) and the numbers of the redundant RBs of all other RPs. Then, the responder checks which of its RPs have surplus RBs, and replies with the indexes of the surplus RPs and RBs. Finally, the requester sends an ACK (Acknowledgment) message to confirm the coordination.

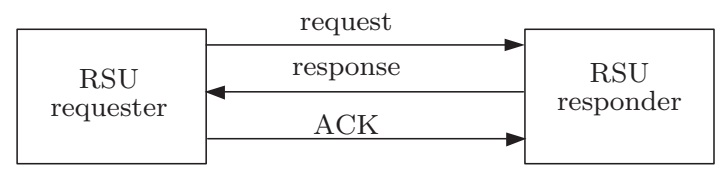

Figure 3 Coordination procedure

To avoid collisions from multiple RSUs proposing coordination at the same time, and to avoid repeated "borrowing" and/or "lending" operations resulting from unordered coordination, we divide the last part of the pre-allocation and coordination phase into 3 sub-stages. We propose the following orderly coordination scheme.

The three sub-stages have indexes $m=0,1,2$. The index of the RSU that proposes coordination in sub-stage $m$ of slot $t$ satisfies

$$
j \bmod 3=(t+m) \bmod 3 .
$$

For example, RSUs 1, 4, and 7 will propose coordination in sub-stage 0 of slot 1 , sub-stage 2 of slot 2 , and sub-stage 1 of slot 3 . RSUs 2,5 , and 8 will propose coordination in sub-stage 1 of slot 1 , sub-stage 0 of slot 2 , and sub-stage 2 of slot 3 . With the order in Eq. (1), different RSUs propose coordination in their corresponding sub-stages in turn, which guarantees that the borrowing requests will not collide with each other. The order of coordination rotates periodically, which guarantees the fairness among the RSUs. Setting the number of sub-stages to 3 is essential to avoid interference among borrowing requests. This is because the interference range $r^{\prime}$ is assumed to be smaller than an RSU's transmission range $R$, and thus vehicles located in RSU $j$ 's coverage will only interfere with the vehicles located in the coverages of
RSUs $j-1$ and $j+1$; the RSUs with an index difference larger than or equal to 3 will not interfere with each other. Therefore, an RSU only needs to coordinate with two immediately adjacent RSUs, and they should have high $(m=0)$, medium $(m=1)$, and low $(m=2)$ priorities when proposing coordination in turn periodically.

It is noted that when using the RBs in $S_{1}$ to $S_{k-1}$, the vehicles located in road segment $k$ of RSU $j$ will only interfere with those located in road segments 1 to $k-1$ of $\operatorname{RSU} j+1$. This is because the distance between road segment $k$ of RSU $j$ and any one of road segments 1 to $k-1$ of RSU $j-1$ is larger than $2 r^{\prime}$, as shown in Fig. 4. Therefore, the decision to "lend" RBs in $S_{1}$ to $S_{k-1}$ should be made only by RSU $j+1$. Similarly, when using the RBs in $S_{k+1}$ to $S_{K}$, the vehicles located in road segment $k$ of RSU $j$ will only interfere with those located in road segments $k+1$ to $K$ of RSU $j-1$, and thus the decision to lend RBs in $S_{k+1}$ to $S_{K}$ should be made only by RSU $j-1$. After receiving the responses from RSUs $j-1$ and $j+1$, RSU $j$ can make a decision as to which RBs to borrow from which RPs, send an ACK to inform RSUs $j-1$ and $j+1$ of its decision, and then move the lent RBs from the lending RPs (e.g., $\left.S_{q}, q=1,2, \cdots, k-1, k+1, \cdots, K\right)$ to the borrowing RPs (e.g., $\left.S_{k}\right)$.

\subsection{BI requests for safety and location tracking accuracy}

The beacon message of vehicle $v$ is denoted as $B_{v}=$ $\left(B A S I C_{v}, I_{v}\right)$, where the basic information vector $B A S I C_{v}=\left(\mathrm{ID}_{v}, L_{v}, w_{v}, \alpha_{v}, h_{v}\right)$, the BI request vector $I_{v}=\left(N_{\mathrm{s}, v}, N_{\mathrm{a}, v}\right)$, and $N_{\mathrm{s}, v}$ and $N_{\mathrm{a}, v}$ are the $\mathrm{BI}$ requests for safety and tracking accuracy, respectively.

The BI requests are calculated according to safety and tracking accuracy requirements. If a vehicle drives at a constant velocity, its neighbors can track it accurately according to the information in its previous beacon message, and therefore the vehicle can stop beaconing for several slots so as to reduce beacon collision. When velocity changes drastically, for 


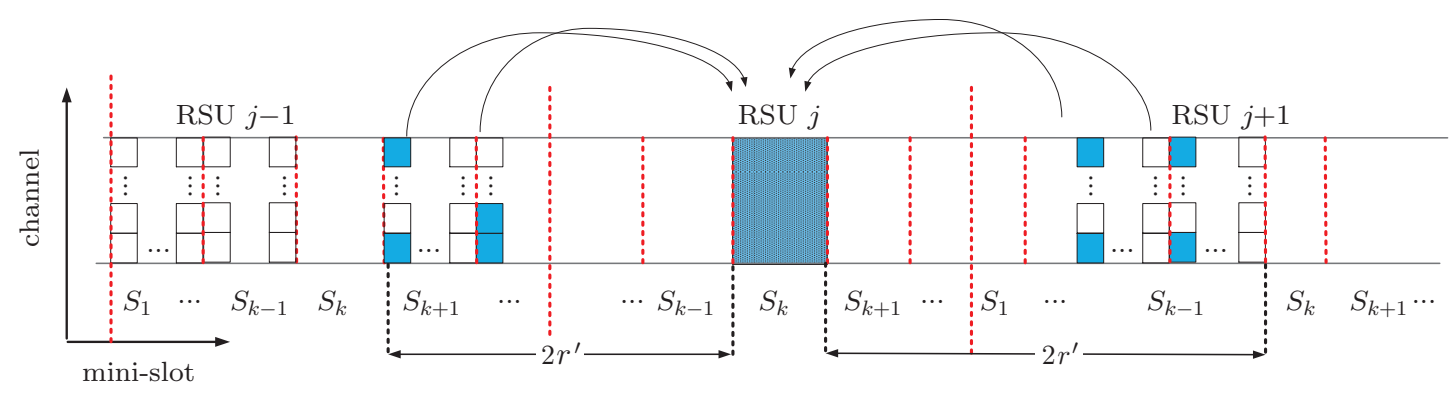

Figure 4 Resource transition sketch.

example, when accelerating or breaking suddenly, the vehicle should reduce its beacon interval to inform its neighbors. In short, the BI requests are based on the vehicle's driving state and environment, and differ between vehicles.

We define the BI request for safety $N_{\mathrm{s}, v}$ as

$$
\begin{aligned}
& N_{\mathrm{s}, v} \\
& \triangleq \begin{cases}1, & T_{\mathrm{H}, v}<T_{\min }, \\
\left\lfloor\frac{N_{0}-1}{T_{\max }-T_{\min }} T_{\mathrm{H}, v}\right. & \\
\left.+\frac{T_{\max }-N_{0} T_{\min }}{T_{\max }-T_{\min }}\right\rfloor, & T_{\mathrm{H}, v} \in\left[T_{\min }, T_{\max }\right], \\
N_{0}, & T_{\mathrm{H}, v}>T_{\max },\end{cases}
\end{aligned}
$$

where time headway $T_{\mathrm{H}, v} \triangleq\left|\left(L_{v^{\prime}}-L_{v}\right) / w_{v}\right|$ is a metric of safety ${ }^{[21]}$. A larger time headway means a smaller probability that a vehicle will crash. $L_{v^{\prime}}$ denotes the location of vehicle $v^{\prime}$ which is located directly in front of vehicle $v . T_{\min }$ and $T_{\max }$ are the minimum and maximum time headways considered, respectively. They are usually obtained by experiments, and determined by the probability of a crash $^{[22]}$. It can be seen from Eq. (2) that $N_{\mathrm{s}, v}=1$ if $T_{\mathrm{H}, v}<T_{\min }$; i.e., when the probability of crash is high, the vehicle should beacon in the next slot. If $T_{\min } \leqslant T_{\mathrm{H}, v} \leqslant T_{\max }, N_{\mathrm{s}, v}$ increases monotonically as $T_{\mathrm{H}, v}$ increases, because a larger $T_{\mathrm{H}, v}$ means a lower probability of a crash and a less urgent request. If $T_{\mathrm{H}, v}>T_{\max }$, the probability of a crash is small, and thus $N_{\mathrm{s}, v}$ can take the maximum allowed BI value $N_{0}$.

We define the BI request for tracking accuracy $N_{\mathrm{a}, v}$ as

$$
N_{\mathrm{a}, v} \triangleq\left\lfloor N_{0}-\left(\frac{N_{0}-1}{\left|\Delta a_{\max }\right|}\right) \cdot|\Delta a|\right\rfloor,
$$

where $|\Delta a|$ is the absolute value of the acceleration difference between the last and current slots. $\left|\Delta a_{\max }\right|$ is the maximum acceleration difference; i.e., the maximum acceleration plus the maximum deceleration. It can be seen that $N_{\mathrm{a}, v}$ equals $N_{0}$ when $|\Delta a|=0$; i.e., beaconing with the maximum allowed BI is sufficient when vehicle $v$ drives at a constant velocity or uniform acceleration. $N_{\mathrm{a}, v}$ decreases as $|\Delta a|$ increases; i.e., more frequent beaconing is required when the acceleration changes more quickly. $N_{\mathrm{a}, v}=1$ when $|\Delta a|=\left|\Delta a_{\max }\right|$; i.e., beaconing should occur in every slot when the acceleration changes sharply.

\subsection{Resource allocation formulation}

Because the vehicles in different road segments utilize different RPs, the resource allocation among different RPs is mutually independent, and can thus be completed separately. Therefore, in the following sections, we study the resource allocation in a road segment in one RSU, if not specified.

As the vehicles drive in and out of a road segment, the beaconing resource requirement changes constantly. When vehicle density is high, the number of vehicles requesting a broadcast of beacon messages in the same slot may be larger than the number of RBs, thus causing collisions. Our goal is to avoid collision by correct resource allocation, which utilizes the difference between BI requests; i.e., allocating the former RBs to the vehicles of which BI requirements are urgent, and the latter RBs to vehicles of which BI requirements are not urgent. Therefore, the $N_{0} C Q$ RBs in the next $N_{0}$ slots should be allocated by jointly considering the BI requests of all 
vehicles, and the number of idle slots for each vehicle since its last beaconing slot. The resource allocation in road segment $k$ (with RP $S_{k}$ ) can be modeled as the following optimization problem

$$
\begin{array}{r}
\text { OP1 : } \max _{X_{n, q, c}^{v}} \sum_{n=1}^{N_{0}} \sum_{q=1}^{Q} \sum_{c=1}^{C} \sum_{v=1}^{V_{k}} U_{v}\left(N_{\text {inter }, v}\right) \cdot X_{n, q, c}^{v} \\
\text { s.t. } X_{n, q, c}^{v}=0, \forall v, \forall n, \text { if }\left|L_{n, v}\right|>R, \\
\sum_{n=1}^{N_{0}} \sum_{q=1}^{Q} \sum_{c=1}^{C} X_{n, q, c}^{v}=1, \forall v, \\
X_{n, q, c}^{v} X_{n, q, c}^{v^{\prime}} \leqslant\left|L_{n, v}-L_{n, v^{\prime}}\right| / 2 r^{\prime}, \forall v^{\prime} \neq v, \\
X_{n, q, c}^{v}=0, \text { if } S_{q, c} \notin S_{k}, \\
X_{n, q, c}^{v} \in\{0,1\},
\end{array}
$$

where indication variable $X_{n, q, c}^{v}=1$ means that $\mathrm{RB}$ $S_{q, c}$ in slot $n$ is allocated to vehicle $v$, otherwise $X_{n, q, c}^{v}=0 . \quad N_{\text {inter }, v}=N_{\text {past }, v}+\sum_{n=1}^{N_{0}} n \cdot X_{n, q, c}^{v}$ represents the number of slots from vehicle $v$ 's last beaconing slot to the next beaconing slot, where $N_{\text {past }, v}$ is the number of slots from vehicle $v$ 's last beaconing slot to the current slot. $\sum_{n=1}^{N_{0}} n \cdot X_{n, q, c}^{v}$ represents the slot index of the RB allocated to vehicle $v$; for example, it equals $n_{1}$ when $X_{n_{1}, q, c}^{v}=1$ $\left(X_{n, q, c}^{v}=0, \forall n \notin n_{1}\right)$, and indicates that the slot index of the RB allocated to vehicle $v$ is $n_{1}$. $L_{n, v}$ denotes the estimated location of vehicle $v$ in slot $n$, which can be derived from the basic information stored in the "vehicle list". $U_{v}\left(N_{\mathrm{inter}, v}\right)$ represents the utility value when the BI value equals $N_{\text {inter }, v}$. Eq. (4) means that the allocation scheme should maximize the sum utility of all vehicles. Eq. (5) means that the RBs in slot $n$ will not be allocated to vehicle $v$ if the vehicle will be out of the RSU's transmission range at that slot. It should be noted that the location data obtained from the global positioning system should be transformed to that in the coordinate system, with its origin be the RSU's location. Eq. (6) means that a vehicle can be allocated only one RB in successive $N_{0}$ slots. Eq. (7) represents the fact that an RB can be allocated to two (or more) vehicles only when the inter-vehicle distance is larger than $2 r^{\prime}$; this is so that the beacons from different vehicles will not collide and interfere in the air. Eq. (8) represents the fact that any RB $S_{q, c}$ in slot $n$ will not be allocated to vehicle $v$ if the $\mathrm{RB}$ is not in RP $S_{k}$.

\subsection{Utility function}

$U_{v}\left(N_{\text {inter }, v}\right)$ in Eq. (4) is the utility value when vehicle $v$ 's BI value equals $N_{\mathrm{inter}, v}$, and is defined as the degree of satisfaction for both safety, and for tracking accuracy. It equals 1 if the allocated RB fully satisfies the two requests, ranges from 0 to 1 if partial satisfaction is achieved with the allocated RB, and equals 0 if $N_{\text {inter, } v}$ is larger than the maximum allowed BI $N_{0}$. So, we define the utility function as

$$
\begin{aligned}
& U_{v}\left(N_{\text {inter }, v}\right) \\
& \triangleq \begin{cases}\beta u_{\mathrm{S}}\left(N_{\text {inter }, v}\right)+ & N_{\text {inter }, v} \leqslant N_{0}, \\
(1-\beta) u_{\mathrm{a}}\left(N_{\text {inter }, v}\right), & N_{\text {inter }, v}>N_{0}, \\
0, & \end{cases}
\end{aligned}
$$

where $\beta$ is a coefficient used to balance safety and the tracking accuracy; this can be set between 0.5 and 1 , because safety is usually more important. $u_{x}\left(N_{\text {inter }, v}\right), x \in\{\mathrm{a}, \mathrm{s}\}$, represents the utility value of safety (if $x=\mathrm{s}$ ) or tracking accuracy (if $x=\mathrm{a}$ ), and is defined as

$$
\begin{aligned}
& u_{x}\left(N_{\mathrm{inter}, v}\right) \\
& \triangleq 1-\frac{1}{N_{0}}\left(\sum_{n=1}^{N_{0}} n X_{n, q, c}^{v}-N_{x \rightarrow \mathrm{p}, v}\right)^{+},
\end{aligned}
$$

where $(*)^{+}=\max (0, *) ; \quad \sum_{n=1}^{N_{0}} n \cdot X_{n, q, c}^{v}$ gives the slot index of the allocated $\mathrm{RB}$ to vehicle $v ; \quad N_{x \rightarrow \mathrm{p}, v}=\left(N_{x, v}-N_{\text {past }, v}\right)^{+}$represents the number of slots which can satisfy the safety (if $x=\mathrm{s}$ ) or tracking accuracy (if $x=\mathrm{a}$ ) request; $\left(\sum_{n=1}^{N_{0}} n \cdot X_{n, q, c}-N_{x \rightarrow \mathrm{p}, v}\right)^{+}$is the number of slots where $N_{\text {inter, } v}$ exceeds $N_{x, v}$. Hence, the ranges of both $u_{x}\left(N_{\text {inter }, v}\right)$ in Eq. (11) and $U_{v}\left(N_{\text {inter }, v}\right)$ in Eq. (10) are $[0,1]$.

The items in Eq. (11) and their relationship are expressed in Fig. 5, and explained as follows. In Fig. 5, each block represents a slot. The left-most green block is the current slot, and its index is 1 . The red block is vehicle $v$ 's previous beaconing slot, 


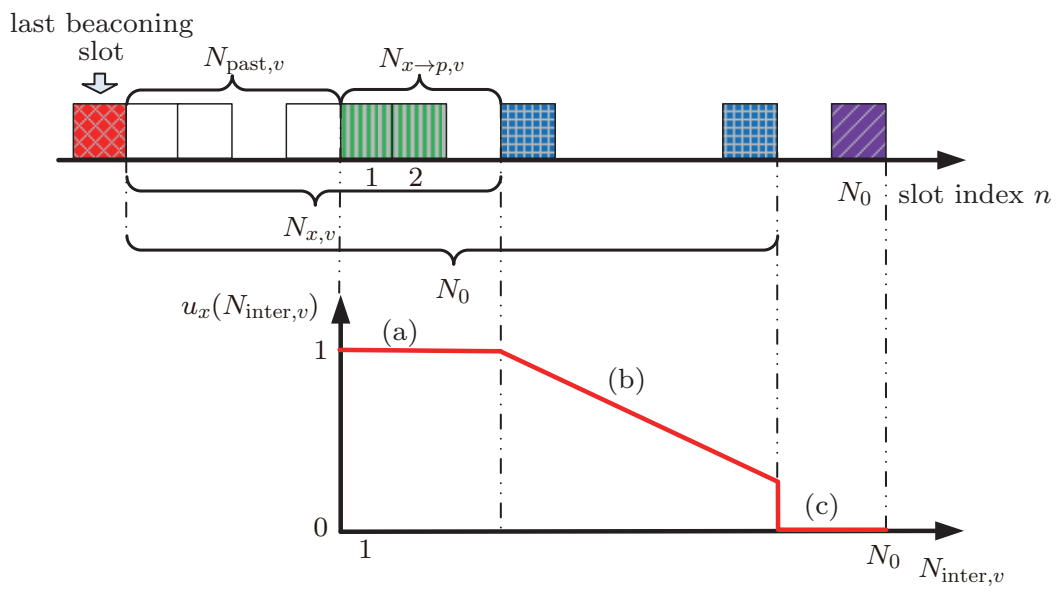

Figure 5 Broadcast slot, broadcast interval, resource allocation, and utility value

and after that $N_{\text {past }, v}$ slots have past. The RBs to be allocated in the current slot involve $N_{0}$ slots with slot indexes $n=1,2, \cdots, N_{0}$. Allocating any green block to vehicle $v$ will satisfy its BI request, because $N_{\text {past }, v}+n_{\text {green }} \leqslant N_{x, v}$, where $n_{\text {green }}$ is the index of a green block. Hence, vehicle $v$ 's utility $u_{x}\left(N_{\text {inter }, v}\right)$ is 1. Allocating any blue block to vehicle $v$ will result in a BI exceeding the requested BI $N_{x, v}$ but by no more than the maximum allowed BI $N_{0}$, and thus the utility value will be in the range $(0,1)$. Allocating any purple block to vehicle $v$ will satisfy neither the requested $\mathrm{BI}$ nor the maximum allowed $\mathrm{BI}$, and the utility value is 0 .

\section{Problem solving}

In this section, we transform the original integer optimization problem OP1 into an MWIS problem in an undirected weighted graph, and propose an algorithm to obtain a resource allocation scheme for adaptive beaconing.

Let $G(Z, E, \omega)$ denote an undirected weighted graph, where $Z$ is the set of vertices, $E$ is the set of edges, and $\omega$ is the vertex weighting set of $G$. We also use $Z(G), E(G)$ and $\omega(G)$ to denote the set of vertices, edges, and vertex weights of $G$, respectively. The MWIS problem is to find a set of vertices $I \subseteq Z(G)$ such that the sum of the weights in $I$ is maximized, and any two vertices $i, j \in I$ have no edge between them. The transformation from OP1 to MWIS is described as follows.

First, we create an undirected un-weighted graph $G^{\prime}\left(Z^{\prime}, E^{\prime}\right)$ as shown in Fig. 6. The set of vertices $Z^{\prime}\left(G^{\prime}\right)$ is of size $N_{0} \times V_{k}$ and consists of the vehicles in the next $N_{0}$ slots. The vertices in the $n$th row represent all of the vehicles in slot $n \in\left[1, N_{0}\right]$, and the vertices in the $i$ th column are the $i$ th vehicle in different slots, where $i \in\left[1, V_{k}\right]$. The set of edges $E^{\prime}\left(G^{\prime}\right)$ contains the edges between the same vehicles in different slots (the blue solid lines), namely $\left\{v_{i}^{n}, v_{i}^{n^{\prime}}\right\} \in$ $E^{\prime}\left(G^{\prime}\right), \forall n \neq n^{\prime}$, and the edges between the vehicles who interfere with each other in the same slot (the dashed red lines), namely $\left\{v_{i}^{n}, v_{j}^{n}\right\} \in E^{\prime}\left(G^{\prime}\right)$. The distance between vehicles $i$ and $j$ is less than $2 r^{\prime}$.

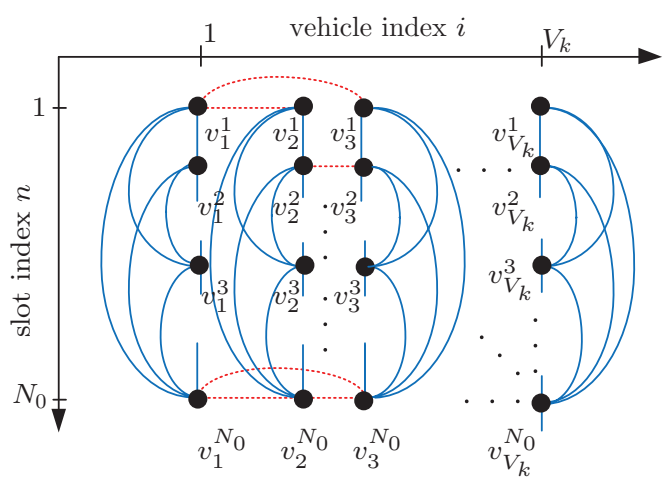

Figure 6 Undirected un-weighted graph $G^{\prime}\left(Y^{\prime}, E^{\prime}\right)$ which reflects the relationship among vehicles 
Next, we construct an undirected weighted graph $G(Z, E, \omega)$ according to $G^{\prime}\left(Z^{\prime}, E^{\prime}\right)$. The vertex set $Z(G)$ is all of the possible combinations of vehicles and RBs, and its size is $N_{0} Q C V_{k}$. The vertex $Z_{n, q, c}^{i} \in Z(G)$ represents the combination of vehicle $i$ and $\mathrm{RB} S_{q, c}$ in slot $n$. Its weight is $\omega\left(Z_{n, q, c}^{i}\right)=$ $U_{i}\left(N_{\text {past }, i}+n\right)$; i.e., the utility value when vehicle $i$ is allocated resource block $S_{q, c}$ in slot $n$, and is thus calculated by substituting $N_{\text {inter }, i}=N_{\text {past }, i}+n$ into Eq. (10). The edge set $E(G)$ of $G(Z, E, \omega)$ contains the edges between any two vehicles which interfere with each other as the result of being allocated the same RB in the same slot, i.e.,

$$
\begin{aligned}
& \left(Z_{n, q, c}^{i}, Z_{n, q, c}^{j}\right) \in E(G), \\
& \quad \text { if }\left\{v_{i}^{n}, v_{j}^{n}\right\} \in E^{\prime}\left(G^{\prime}\right) \text { and } i \neq j,
\end{aligned}
$$

and also contains the edges between the same vehicle being allocated RBs in different slots, i.e.,

$$
\begin{aligned}
& \left(Z_{n, q, c}^{i}, Z_{n^{\prime}, q, c}^{i}\right) \in E(G), \\
& \quad \text { if }\left\{v_{i}^{n}, v_{i}^{n^{\prime}}\right\} \in E\left(G^{\prime}\right) \text { and } n \neq n^{\prime} .
\end{aligned}
$$

Note that Eq. (12) reflects Eq. (7); i.e., the same RB should be allocated to vehicles that do not interfere with each other. Eq. (13) reflects Eq. (6) and implies that a vehicle can be allocated one RB in successive $N_{0}$ slots. Solving OP1 is transformed into finding an MWIS $I \in Z(G)$, and indicator $X_{n, q, c}^{i}=1$ if vertex $Z_{n, q, c}^{i} \in I$, otherwise it equals 0 .

We propose an ABI (Adaptive Beacon Interval) algorithm by adopting the GWIN2 algorithm which is one of the low-complexity algorithms for solving MWIS problems ${ }^{[23]}$. Let $N_{G}\left(Z_{n, q, c}^{i}\right)$ denote the set of neighbor vertices of vertex $Z_{n, q, c}^{i}$, and $N_{G}^{+}\left(Z_{n, q, c}^{i}\right)=N_{G}\left(Z_{n, q, c}^{i}\right) \cup\left\{Z_{n, q, c}^{i}\right\}$ represent the set of vertex $Z_{n, q, c}^{i}$ and its neighbor vertices. In the ABI algorithm, an empty set $I$ and an undirected weighted graph $G_{1}:=G$ are set up in the initialization phase. In the $j$ th $(j=1,2 \cdots)$ iteration, randomly select a vertex $Z_{n, q, c}^{i}$ from $G_{j}$; if it is the vertex of which $\omega\left(Z_{n, q, c}^{i}\right) / \sum_{z \in N_{G}^{+}\left(Z_{n, q, c}^{i}\right)} \omega(z)$ is the maximum among all vertices of $N_{G}^{+}\left(Z_{n, q, c}^{i}\right)$, then put it into $I$; obtain graph $G_{j+1}$ by deleting $N_{G}^{+}\left(Z_{n, q, c}^{i}\right)$ from $G_{j}$. The iteration continues until no vertex is left in graph $G_{j}$. Finally, let $X_{n, q, c}^{i}=1$ if $Z_{n, q, c}^{i} \in I$, otherwise $X_{n, q, c}^{i}=0$. The ABI algorithm is summarised in algorithm below.

In the worst case, the computational complexity of the ABI algorithm is $O\left(N_{0} Q C V_{k}\right)$. This occurs when all vertices in $G(Z, E, \omega)$ are independent, and thus altogether $N_{0} Q C V_{k}$ vertices should be put into the MWIS.

The ABI algorithm is performed by the RSU at the beginning of each slot, and the obtained resource allocation scheme is for the current and next $N_{0}-1$ slots. It should be noted that in the obtained allocation scheme, only the part for the current slot is executed; i.e., only the RBs in the current slot are allocated to part of the vehicles, and the other part of the scheme will not be applied. The reason is as follows. The information in the "vehicle list" changes slot by slot because there may be vehicles driving in and out of the RSU's transmission range; this changes the vehicle numbers and BI requests, and because the vehicles which are allocated an RB in the current slot will broadcast their new $\mathrm{BI}$ requests; this changes the priorities of all vehicles. Therefore, resource allocation should be performed for each slot according to the latest information in the "vehicle list".

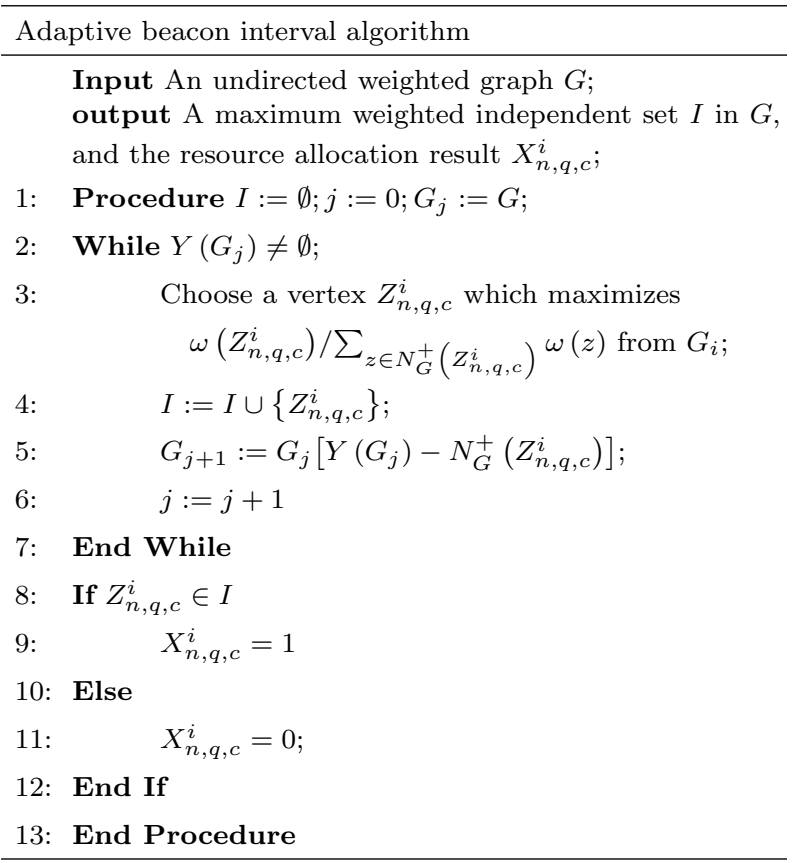




\section{Simulation results}

In this section we perform simulations to validate the effectiveness of the proposed ABIwRC (Adaptive Beaconing Interval with Resource Coordination) method, and compare this with two baseline algorithms.

\subsection{Simulation settings}

We set up a highway $4000 \mathrm{~m}$ in length. The vehicles' maximum acceleration is $2.6 \mathrm{~m} / \mathrm{s}^{2}$, and the maximum deceleration is $4.5 \mathrm{~m} / \mathrm{s}^{2}$, thus $\left|\Delta a_{\max }\right|=7.1$ $\mathrm{m} / \mathrm{s}^{2}$. The deviation threshold $\eta$ is $0.5 \mathrm{~m}$, and the maximum and minimum time headways $T_{\max }$ and $T_{\min }$ are set to $10 \mathrm{~s}$ and $1.5 \mathrm{~s}$, respectively ${ }^{[22,24,25]}$. The vehicle densities are $[40,50,60,80,100,120]$ veh $/ \mathrm{km}$, which cover a light load, medium load, and heavy load. A light load means that the resource is sufficient to satisfy the safety and tracking accuracy requirements of all vehicles, while a heavy load means that some vehicle requests cannot be satisfied. The highway and vehicle traces are created by $\mathrm{SUMO}^{[26]}$. For each vehicle density, 15 random traces are created with $N_{\text {simu }}=900$ slots per trace, and the simulation results presented were obtained by averaging over the 15 traces. The transmission range of an RSU, $R$, is $150 \mathrm{~m}$. The transmission range between vehicles, $r$, is $100 \mathrm{~m}$, and for simplicity the interference range, $r^{\prime}$, is set equal to $r$.

Similar to the IEEE 1609.4 protocol $^{[27]}$, in the simulation we set one single dedicated channel to support the beaconing service. The bandwidth is 10 $\mathrm{MHz}$, and the transmission rate is $2 \mathrm{Mbit} / \mathrm{s}$. The slot duration $T_{\text {slot }}$ is $100 \mathrm{~ms}$, and there are 17 minislots per slot. The length of the beacon message $F$ is $6400 \mathrm{bits}$, and the duration of the maximum allowed BI $T_{0}$ is $1 \mathrm{~s}^{[20]}$, and so $N_{0}=10$. The weight coefficient $\beta$ is 0.8 .

\subsection{Baseline}

Two baseline algorithms are compared in the simulation. First, the ABRC (Adaptive Beacon Rate
Control) algorithm in Ref. [10] is adopted as a baseline algorithm. The goal of the ABRC algorithm is to guarantee tracking accuracy. Of the existing algorithms, it is the closest to the proposed method. In the ABRC algorithm, a vehicle estimates its location from the information in its last beacon message. If the deviation between its actual and estimated location is larger than the threshold, a new beacon message is needed to inform neighbors of its current state. The vehicle will randomly choose an $\mathrm{RB}$ in the current slot and broadcasts; otherwise, it keeps silent. Another baseline is the ABI (Adaptive Beaconing Interval) method proposed in Ref. [28], in which the RSUs do not divide the road into road segments, and will not coordinate with adjacent RSUs on RPs. To emphasize the difference between it and the ABIwRC method in this paper, for our purposes we name it the ABI-NC (Adaptive Beaconing Interval with No resource Coordination) algorithm.

\subsection{Performance metrics}

To evaluate the performance of the ABIwRC method and the two baseline algorithms, the following metrics are adopted.

(1) BRR (Beacon Reception Ratio): BRR is defined as $\left(N_{\text {simu }} V_{n}\right)^{-1} \sum_{n=1}^{N_{\text {simu }}} \sum_{v=1}^{V_{n}} O_{n, \text { succ }}^{v} / O_{n, \text { all }}^{v}$, where $V_{n}$ is the total number of vehicles broadcasting beacon messages in slot $n$. $O_{n, \text { all }}^{v}$ and $O_{n, \text { succ }}^{v}$ are the number of all neighbors of vehicle $v$ in slot $n$, and the number of neighbors that receive the beacon message successfully, respectively. BRR is averaged on all vehicles within all RSU road segments, and can therefore reflect the degree of collision. A higher BRR means a lower degree of beacon collision, and a more effective guarantee of safety.

(2) RA (Ratio of Accuracy): RA is defined as $\left(N_{\text {simu }} V_{n}\right)^{-1} \sum_{n=1}^{N_{\text {simu }}} \sum_{v=1}^{V_{n}} O_{n, \text { accu }}^{v} / O_{n, \text { all }}^{v}, \quad$ where $O_{n \text {,accu }}^{v}$ represents the number of neighbor vehicles that can accurately track vehicle $v$. RA is also averaged on all vehicles located within all RSU road segments.

(3) RS (Ratio of Safety): RS is defined as ( $N_{\text {simu }}$ $\left.\times V_{n}\right)^{-1} \sum_{n=1}^{N_{\text {simu }}} \sum_{v=1}^{V_{n}} \operatorname{sgn}\left(N_{\mathrm{s}, v}-N_{\text {inter }, v}\right) \times \mathrm{BRR}$, 
where $\operatorname{sgn}\left(N_{\mathrm{s}, v}-N_{\text {inter }, v}\right)$ equals 1 when $N_{\mathrm{s}, v}-$ $N_{\text {inter, } v} \geqslant 0$; i.e., the safety of vehicle $v$ can be guaranteed, otherwise $\operatorname{sgn}\left(N_{\mathrm{s}, v}-N_{\text {inter }, v}\right)$ equals 0 . $\operatorname{sgn}\left(N_{\mathrm{s}, v}-N_{\text {inter }, v}\right) \times \mathrm{BRR}$ is the probability that vehicle $v$ broadcasts its beacon message satisfying $N_{\mathrm{s}, v}$ and that the message is received.

(4) CR (Coordination Ratio): CR is defined as $N_{\text {cor }} / N_{\text {simu }}$, where $N_{\text {cor }}$ is the number of coordination attempts among RSUs. $N_{\text {cor }}$ will increase by one when the number of mini-slots of any RP is insufficient and the RSU starts a coordination procedure.

\subsection{Performance}

To evaluate the effect of the driving state on $N_{\mathrm{s}, v}$ and $N_{\mathrm{a}, v}$, Fig. 7 shows the velocity, distance, time headway, $N_{\mathrm{s}, v}$ and $N_{\mathrm{a}, v}$, and distance deviation of a vehicle, in 150 successive slots. Fig. 7(a) shows the velocities of the rear vehicle, a vehicle directly in front of it (the left vertical axis), and the distance between them (the right vertical axis). The velocities decrease at first, and then increase. This means that the two vehicles reach a traffic light, stop one after the other, and then accelerate to leave the intersection. The distance between them first decreases and then increases. This is because the front vehicle reaches the intersection first and decreases in velocity, and so the rear vehicle gets closer. After the traffic light turns green, the front vehicle accelerates first and increases the distance. Fig. 7(b) shows the changes in time headway (the left vertical axis) and $N_{\mathrm{s}, v}$ (the right vertical axis) during the above situation. In slots 0-95, although the distance between vehicles decreases, the decrease in the rear vehicle's velocity leads to the increase in time headway. In slots 95-150, the increase in the rear vehicle's velocity leads to the rapid decrease in time headway. Meanwhile, $N_{\mathrm{s}, v}$ increases and then decreases along with the time headway, and can thus reflect the safety request.

Fig. 7(c) shows the rear vehicle's $N_{\mathrm{a}, v}$ (the right vertical axis) and the distance deviation after $N_{\mathrm{a}, v}$ slots (the left vertical axis). It can be observed from Fig. 7(c) that the distance deviation is gener- ally lower than the threshold (dashed red line). It is larger than the threshold only around slot 100 .

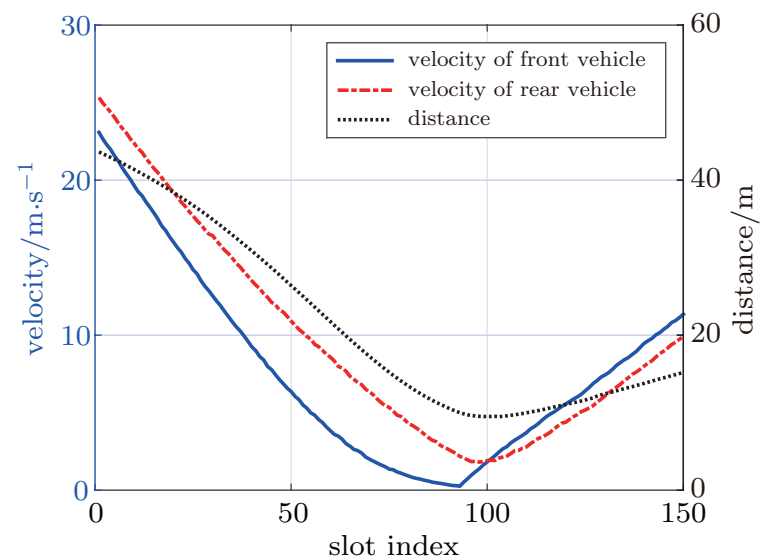

(a)

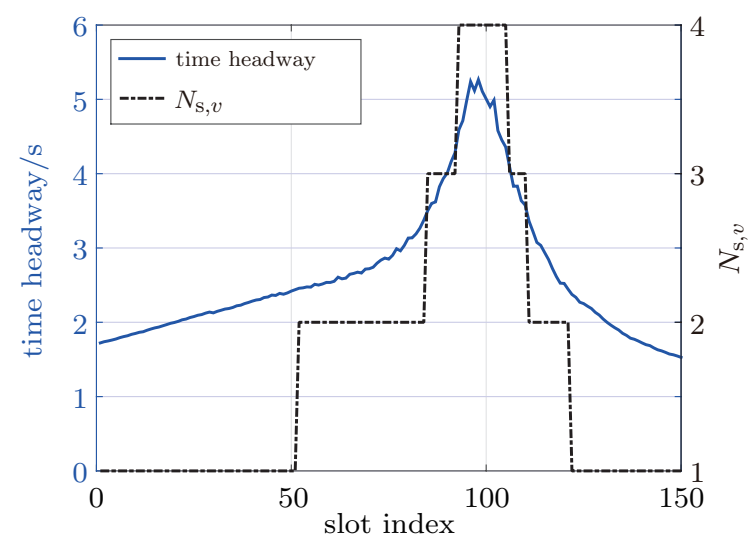

(b)

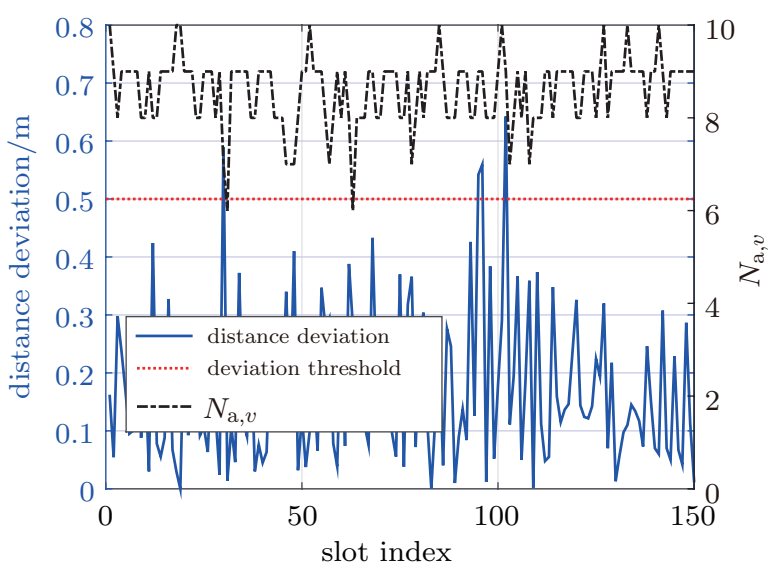

(c)

Figure 7 Velocity, distance, time headway, $N_{\mathrm{s}, v}$ and $N_{\mathrm{a}, v}$ as well as the distance deviation of the rear vehicle (100 veh $/ \mathrm{km})$. (a) Velocities and inter-vehicle distance; (b) time headway and $N_{\mathrm{s}, v}$ of rear vehicle; (c) rear vehicle's $N_{\mathrm{a}, v}$, and the distance deviation 


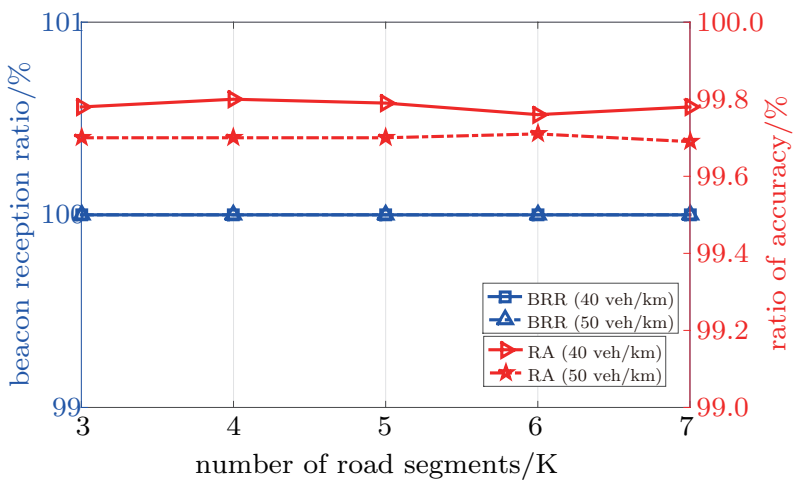

(a)

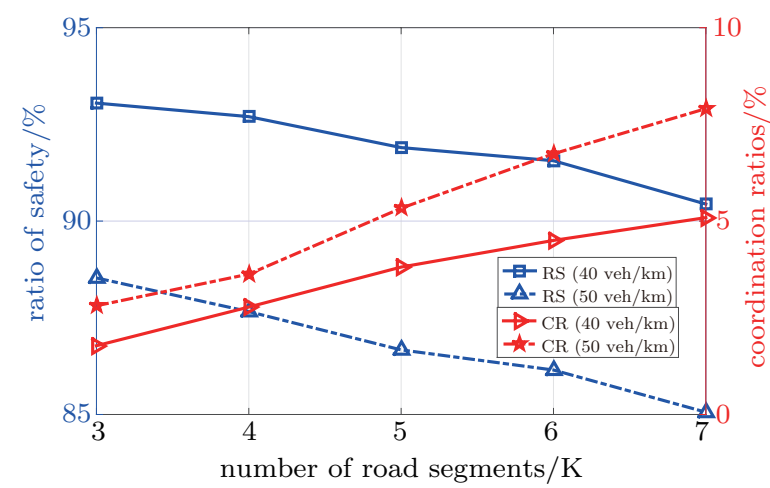

(b)

Figure 8 Effect of number of road segments $K$ on BRR, RA, RS, and CR among RSUs (40 and 50 veh/km). (a) BRR and RA; (b) RS and CR

It can be seen from Fig. 7(a) that around slot 100 the traffic light turns green, and the vehicle velocity increases suddenly. This sudden change cannot be predicted from historical information, and thus causes the distance deviation to be higher than the threshold. In the simulation, the average distance deviation is actually $0.1716 \mathrm{~m}$, and $96.34 \%$ of the deviation threshold is lower than the threshold. These results outperform those of a recent study ${ }^{[29]}$, which adapts the beacon frequency to achieve tracking accuracy.

Fig. 8 shows the effect of the number of road segments $K$ on BRR, RA, RS, and $\mathrm{CR}$, under vehicle densities of 40 and $50 \mathrm{veh} / \mathrm{km}$. Fig. 8(a) shows the changes in BRR (the blue line corresponding to the left vertical axis) and RA (the red line corresponding to the right vertical axis). It can be observed that as $K$ increases, the BRRs under both vehicle densities are $100 \%$. This means that $K \geqslant 3$ is sufficiently large that there are no beacon collisions among the vehicles on the road segments of adjacent RSUs. It can also be seen that as $K$ increases, the RAs under both vehicle densities remain almost unchanged. It is easier to satisfy BI request $N_{\mathrm{a}, v}$ as compared with $N_{\mathrm{s}, v}$, and thus RA is usually large and not sensitive to the value of $K$.

Fig. 8(b) shows the changes in RS (the blue line corresponding to the left vertical axis) and CR (the red line corresponding to the right vertical axis). It can be observed that the higher the vehicle density, the higher the CR and the lower the RS. The reason for this is that a higher vehicle density means that there are more vehicles in a road segment; this means a shortage of RBs is more likely, which leads to more resource coordination and a degradation in performance of RA. It can also be seen that a larger number of road segments $K$ results in a higher CR and lower RS. This is because a larger $K$ means more RPs, and fewer RBs in each RP. Therefore, the probability that the number of RBs cannot meet the vehicles' requests increases, which leads to more resource coordination. A larger $K$ means shorter road segments, which leads to more frequent inter-segment handover, and thus leads to a lower RS.

Fig. 9 shows the effect of vehicle density on BRR, $\mathrm{RA}$, and RS. The comparison is made with the proposed ABIwRC method and ABI-NC algorithm, as well as with the ABRC algorithm. Fig. 9(a) shows the BRRs versus vehicle density. It can be observed that as vehicle density increases, the BRRs of ABIwRC method remain invariant. This is because collisions of beacon messages among vehicles located in different RSUs are avoided through partition of road segments. The BRRs of the ABI-NC algorithm and $\mathrm{ABRC}$ algorithm decrease as the vehicle density increases. This is because the ABI-NC algorithm cannot avoid beacon message collisions among the vehicles located in different RSUs, and the ABRC algorithm allocates resources without overall consideration; thus, this algorithm cannot avoid beacon 
message collisions among the vehicles located within the adjacent and/or the same RSU.

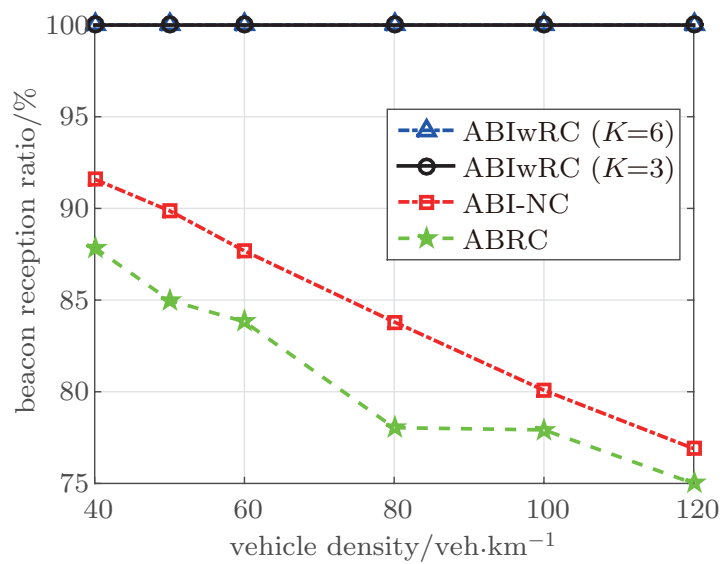

(a)

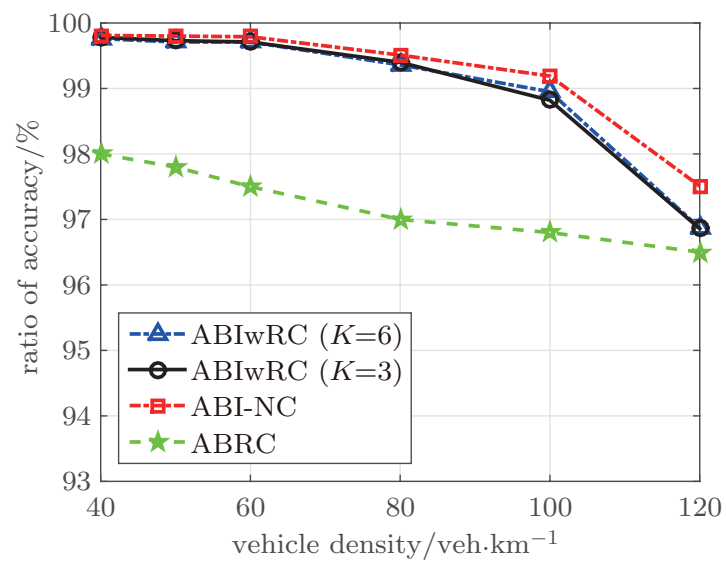

(b)

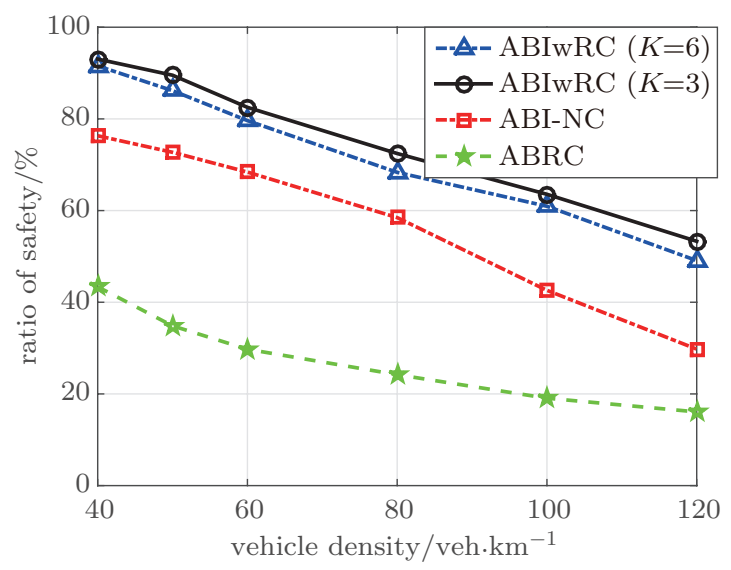

(c)

Figure 9 BRR, RA, and RS vs vehicle density. (a) Beacon reception ratio; (b) ratio of accuracy; (c) ratio of safety
It can be observed from Fig. 9(b) that as vehicle density increases, the RAs of all algorithms decrease. This is because a higher vehicle density means more vehicles competing for resources at the same time, and thus this decreases each vehicle's probability of being allocated RBs. The RAs of the ABIwRC method with $K=3$ and $K=6$ are almost the same, because RA is not sensitive to the number of road segments $K$, as shown in Fig. 8(a).

Fig. 9(c) shows the RS versus the vehicle density. It can be observed that the RSs of all algorithms decrease as the vehicle density increases, but the proposed ABIwRC method has higher RSs than both of the baseline algorithms. The RSs of the ABIwRC method with $K=3$ are better than those with $K=6$. This is because a larger $K$ leads to frequent inter-segment handover, and harms the performance of the ABIwRC method. The RSs of the ABIwRC method with $K=3$ and $K=6$ are higher than those of the ABI-NC algorithm, because the ABINC algorithm has lower BRRs, which negatively impact safety. Most importantly, the conclusion can be drawn that the lowest $K$ which does not introduce interference is optimal; i.e., $K=3$ in our simulation.

Comparing Figs. 9(b) and 9(c), it can be observed that RS decreases more than RA. This is because as the vehicle density increases, the distances between vehicles get shorter, and it is easier for vehicles to crash; this usually makes $N_{\mathrm{s}, v}$ smaller than $N_{\mathrm{a}, v}$, so $N_{\mathrm{s}, v}$ is much more difficult to satisfy than $N_{\mathrm{a}, v}$.

\section{Conclusion}

In this paper, an adaptive beaconing method in vehicular networks is proposed. Taking a vehicle's driving safety and location tracking accuracy into consideration, each vehicle broadcasts its BI requests. The RSU allocates RBs after necessary resource coordination so as to maximize the sum utility by avoiding beacon collision, and so satisfies the BI requests of all vehicles. Simulation results show that the proposed method outperforms the baseline algorithms in terms of beacon reception ratio, ratio of location tracking accuracy, and ratio of vehicle driving safety. The 
proposed method is designed for highway scenarios where RSUs are deployed with a line topology. It can be extended to urban environments, however in this case each RSU can have more than two adjacent RSUs; this makes the resource coordination among RSUs much more complicated, and needs further investigation.

\section{References}

[1] H. B. Zhou, B. Liu, F. Hou, et al. Spatial coordinated medium sharing: optimal access control management in Drive-Thru Internet [J]. IEEE trans. intell. transp. syst., 2015, 16(5): 2673-2686.

[2] K. Ota, M. Dong, S. Change, et al. MMCD: cooperative downloading for highway VANETs [J]. IEEE trans. emerg. top. comput, 2015, 3(1): 34-43.

[3] H. L. He, H. G. Shan, A. P. Huang, et al. Resource allocation for video service in heterogeneous cognitive vehicular networks [J]. IEEE trans. veh. technol., 2016, 65(10): 7917-7930.

[4] L. Sun, H. G. Shan, A. P. Huang, et al. Channel allocation for adaptive video streaming in vehicular networks [J]. IEEE trans. veh. technol., 2017, 66(1): 734-747.

[5] H. B. Zhou, B. Liu, T. H. Luan, et al. Chaincluster: engineering a cooperative content distribution famework for highway vehicular communications [J]. IEEE trans. intell. transp. syst., 2014, 15(6): 2644-2657.

[6] H. T. Cheng, H. G. Shan, W. H. Zhuang. Infotainment and road safety service support in vehicular networking: from a communication perspective $[\mathrm{J}]$. Mechanical systems and signal processing, 2011, 25(6): 2020-2038.

[7] Y. G. Bi, H. G. Shan, X. M. Shen, et al. A multi-hop broadcast protocol for emergency message dissemination in urban vehicular Ad hoc networks [J]. IEEE trans. intell. transp. syst., 2016, 17(3): 736-750.

[8] Institute of Electrical and Electronics Engineers Std 1690 Working Group. 1609.2-IEEE standard for wireless access in vehicular environments-security services for applications and management messages [S]. 2013: 3.

[9] European Telecommunications Standards Institute. Intelligent transportation systems (ITS); vehicular communications; basic set of applications; part 2: specification of cooperative awareness basic service [S]. 2013: 4.

[10] H. Nguyen, H. Jeong. Adaptive beacon rate control algorithm for vehicular Ad hoc networks [C]//IEEE International Conference on Ubiquitous and Future Networks, DA NANG, Vietnam, 2013: 652-653.

[11] G. Kim, S. Lee, H. Park, et al. A request based adaptive beacon rate control scheme for vehicular Ad hoc networks [C]//IEEE International Conference on Ubiquitous and Future Networks, Vienna, Austria, 2016: 67-
69.

[12] A. Hassan, M. H. Ahmed, M. A. Rahman. Adaptive beaconing system based on fuzzy logic approach for vehicular network [C]//IEEE Symposium on Personal, Indoor, and Mobile Radio Communications, London, UK, 2013: 2581-2585.

[13] M. M. Alotaibi, H. T. Mouftah. Adaptive expiration time for dynamic beacon scheduling in vehicular Ad hoc networks [C]//IEEE Vehicular Technology Conference, Boston, USA, 2015: 1-6.

[14] W. T. Zhu, D. Y. Gao, C. H. Foh, et al. A collision avoidance mechanism for emergency message broadcast in urban VANET [C]//IEEE Vehicular Technology Conference, Nanjing, China, 2016: 1-5.

[15] A. Hassan, W. H. Zhuang, L. Li. VeMAC: a TDMAbased MAC protocol for reliable broadcast in VANETs [J]. IEEE trans. mob. comput., 2013, 12(9): 1724-1736.

[16] J. Sahoo, E. H. K. Wu, P. K. Sahu, et al. Congestioncontrolled-coordinator-based MAC for safety-critical message transmission in VANETs [J]. IEEE trans. intell. transp. syst., 2013, 14(3): 1423-1437.

[17] S. R. Kolte, M. S. Madankar. Adaptive congestion control for transmission of safety messages in VANET [C]//International Conference for Convergence of Technology, Pune, India, 2014: 1-5.

[18] A. J. Muhammad, T. N. Duy, Y. K. Jamil. Distributed spatial reuse distance control for basic safety messages in SDMA-based VANETs [J]. Vehicular communications, 2015, 2(1): 27-35.

[19] J. A. Pienaar. Random networks for communication: from statistical physics to information systems [M]. UK: Cambridge University Press, 2007.

[20] CAMP Vehicle Safety Communications Consortium. Vehicle safety communications project: task 3 final report: identify intelligent vehicle safety applications enabled by DSRC [R], 2005.

[21] K. Vogel. A comparison of headway and time to collision as safety indicators $[\mathrm{J}]$. Accident analysis \& prevention, 2003, 35(3): 427-433.

[22] T. J. Ayres, L. Li, D. Schleuning, et al. Preferred time-headway of highway drivers [C]//IEEE Intelligent Transportation Systems, Oakland, USA, 2001: 826-829.

[23] S. Sakai, M. Togasaki, K. Yamazaki. A note on greedy algorithms for the maximum weighted independent set problem [J]. Discrete applied mathematics, 2003, 126(23): 313-322.

[24] A. J. Muhannad, Y. K. Jamil. Performance analysis of a time headway based rate control algorithm for VANET safety applications [C]//International Conference on Signal Processing and Communication Systems, Carrara-Gold Coast, Australia, 2013: 1-6.

[25] T. M. Meirav, S. David. Minimum and comfortable driving headways: reality versus perception $[\mathrm{J}]$. Human factors, 2001, 43(1): 159-172.

[26] M. Behrisch, L. Bieker, D. krajzew. Simulation 
of urban mobility [EB/OL]. https://sourceforge.net/ projects/sumo/.

[27] IEEE Std 1690 Working Group. 1609.4-IEEE standard for wireless access in vehicular environments (WAVE) -multi-channel operation [S]. New York, 2011: 7.

[28] L. Sun, A. P. Huang, H. G. Shan, et al. Adaptive beaconing for collision avoidance and tracking accuracy in vehicular networks [C]//IEEE Wireless Communications and Networking Conference, San Francisco, USA, 2017: $1-6$

[29] X. H. Xiang, W. H. Qin. Movement-based beacon rate control for vehicle-to-vehicle communications [C]//International Conference on Transportation Information and Safety, Wuhan, China, 2015: 190-194.

\section{About the authors}

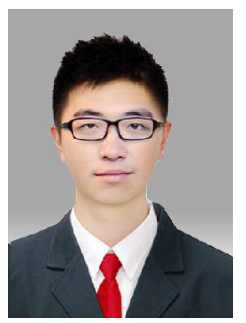

Long Sun received the B.Sc. degree in information engineering from Zhejiang University, Hangzhou, China, in 2012. He is currently working toward the Ph.D. degree with the Institute of Information and Communication Engineering, Zhejiang University. His current research interests include video streaming in vehicular Ad hoc networks and quality-of-service provisioning in cognitive radio networks. (Email: anyuping@yeah.net)

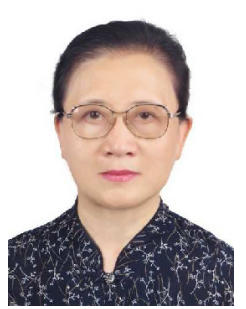

Aiping Huang graduated from Nanjing Institute of Post and Telecommunications, Nanjing, China, in 1977, received the M.S. degree from Nanjing Institute of Technology (Southeast University), Nanjing, in 1982, and the Licentiate of Tech. degree from Helsinki University of Technology (HUT), Espoo, Finland, in 1997. From 1977 to 1980, she was an engineer with the Design and Research Institute of Post and Telecommunications Ministry, China. From 1982 to 1994, she was initially an assistant professor and then an associate professor with Zhejiang University (ZJU), Hangzhou, China. From 1994 to 1998, she was a visiting scholar and a research scientist with HUT (Aalto University). Since 1998, she has been a full professor with ZJU. She is currently with the Zhejiang Provincial Key Laboratory of Information Processing and Communication Networks, Hangzhou. She has authored a book and more than 190 papers in refereed journals and conferences on communications and networks and signal processing. Her current research interests include heterogeneous networks, performance analysis and cross-layer design, and planning and optimization of cellular mobile communication networks.

Dr. Huang serves as a Vice-Chair of the IEEE ComSoc Nanjing Chapter. (Email: aiping.huang@zju.edu.cn)

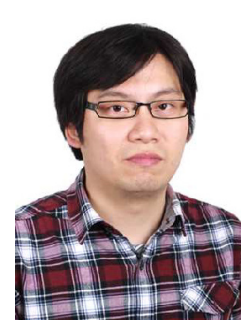

Hangguan Shan [corresponding author] received the B.Sc. degree in electrical engineering from Zhejiang University, Hangzhou, China, in 2004, and the $\mathrm{Ph} . \mathrm{D}$. degree in electrical engineering from Fudan University, Shanghai, China, in 2009. From 2009 to 2010, he was a Post-Doctoral Research Fellow with the University of Waterloo, Waterloo, ON, Canada. Since 2011, he has been with the College of Information Science and Electronic Engineering, Zhejiang University, where he is currently an associate professor. He is also currently with the Zhejiang Provincial Key Laboratory of Information Processing and Communication Networks, Hangzhou. His current research focuses on cross-layer protocol design, resource allocation, and the quality-of-service provisioning in wireless networks.

Dr. Shan was a co-recipient of the Best Industry Paper Award from the 2011 IEEE WCNC, Quintana Roo, Mexico. He is currently an editor of the IEEE Transactions on Green Communications and Networking. He has served on the Technical Program Committee as a member in various international conferences, including the IEEE Globecom, the ICC, the WCNC, and the VTC. He serves as the Track Leading Co-Chair of Future Trends and Emerging Technologies Track of the IEEE VTC 2017-Fall and has served as a Track CoChair of Green Communications and Networks Track of the IEEE VTC 2016-Fall. He also served as the Publicity CoChair for the third and fourth IEEE International Workshop on Wireless Sensor, Actuator, and Robot Networks and the fifth International Conference on Wireless Communications and Signal Processing. (Email: hshan@zju.edu.cn)

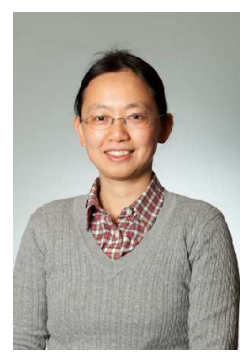

Lin Cai received her M.A.Sc. and Ph.D. degrees in electrical and computer engineering from the University of Waterloo, Waterloo, Canada, in 2002 and 2005, respectively. Since 2005, she has been with the Department of Electrical \& Computer Engineering at the University of Victoria, and she is currently a professor. Her research interests span several areas in communications and networking, with a focus on network protocol and architecture design supporting emerging multimedia traffic over wireless, mobile, Ad hoc, and sensor networks.

She has been a recipient of the NSERC Discovery Accelerator Supplement Grants in 2010 and in 2015, and the Best Paper Awards of IEEE ICC 2008 and IEEE WCNC 2011. She has served as a TPC symposium co-chair for IEEE Globecom'10 and Globecom'13, and the associate editor for IEEE transactions on wireless communications, IEEE transactions on vehicular technology, EURASIP journal on wireless communications and networking, International journal of sensor networks, and Journal of communications and networks (JCN). (Email: cai@ece.uvic.ca) 